\title{
Análisis de calidad de agua de la quebrada El Horno en la colonia Nueva Esperanza, Danlí, Departamento El Paraíso
}

\author{
Yefrin Valladares ${ }^{1}$ \\ Alba Osorto ${ }^{2}$ \\ Alicia Lanza $^{3}$ \\ Jairo Martínez ${ }^{4}$
}

\section{RESUMEN}

Una de las principales preocupaciones a nivel mundial en los últimos años ha sido los cuerpos de agua superficiales y más grande aun, la calidad del agua ya que son la principal fuente de abastecimiento de agua de uso humano. La colonia Nueva Esperanza, es una de las colonias más populosas del departamento de El Paraíso con una gran cantidad de pobladores, de las cuales gran parte no cuenta con el total de los servicios básicos y primordialmente agua potable 0 un flujo constante y aguas negras. En esta colonia se encuentra la quebrada El Horno, la cual tiene una extensión de $4.72 \mathrm{Km} 2$ y es una fuente de agua para la población. El análisis de la calidad del agua de la quebrada El Horno se realizó entre los meses de septiembre a noviembre del 2016. Se establecieron tres puntos de muestreo a lo largo de dicha quebrada, se tomaron muestras para el análisis fisicoquímico, muestras microbiológicas y biológicas. Cada uno de los análisis se realizó en, en los laboratorios de la UNAH-TEC Danlí. Uno de los elementos químicos (aluminio) presento concentraciones elevadas de $0.317 \mathrm{mg} / \mathrm{l}$, pues según normativa de la Secretaria de Salud supera su rango permitido de $0.20 \mathrm{mg} / \mathrm{l}$. La cantidad de coliformes encontrados tanto fecales y totales en cada uno de los muestreos en los tres puntos fue incontable. Para los análisis biológicos, el IBF-SV2010 fue el índice que presentó un dato más certero ya que confirmó lo que los demás análisis mostraron: la contaminación de la quebrada es evidente. El principal problema que se presenta en la colonia son las tuberías de pozos sépticos y los mismos pozos sin el recubrimiento adecuado ya que estos pobladores no cuentan con conexión al alcantarillado sanitario.

Palabras clave: Interdisciplinariedad, Ingeniería, Vinculación, Investigación, capacidades

\footnotetext{
${ }^{1}$ Autor, Beneficiario de beca de estudiante de posgrado de la DICYP-UNAH. Profesor de biología en UNAH-TEC Danlí

${ }^{2}$ Coautora, Profesora de química en UNAH-TEC Danlí: alba.osorto@unah.edu.hn

${ }^{3}$ Coautora, Profesora de química en UNAH-TEC Danlí: alicia.lanza@unah.edu.hn

${ }^{4}$ Asesor, Profesor de informática en UNAH-TEC Danlí: jairo.matinez@unah.edu.hn
} 


\section{ABSTRACT}

One of the main concerns worldwide in recent years has been the surface water bodies and even more, the quality of the water since they are the main source of water for human use. The neighborhood in Nueva Esperanza is one of the most populous colonies in the department of El Paraíso with a large number of inhabitants, of which a large part does not have the total of basic services such as potable wáter, constant flow of water and a sewage system. The quebrada El Horno, is a source of water for the population and it has an extension of $4.72 \mathrm{Km} 2$. The water quality analysis of the quebrada El Horno was carried out between the months of September to November 2016. Three sampling points were established along the creek, microbiological and biological samples were taken for physicochemical analysis. Each of the analysis was carried out in the laboratories of the UNAH-TEC Danlí. One of the chemical elements (aluminum) showed high concentrations of $0.317 \mathrm{mg} / \mathrm{I}$, because according to the regulations of the Ministry of Health exceeds its permitted range of $0.20 \mathrm{mg} / \mathrm{l}$. The amount of coliforms found both in fecal and total análisis in each three points of the samples was uncountable. For the biological analyzes, the IBF-SV2010 was the index that presented more accurate data since it confirmed what the other analyzes showed: the contamination of the stream is evident The main problem that occurs in the colony are septic well pipes. These wells were constructed without the adequate coating because the lack connection to the sanitary sewer system.

Keywords: surface water, water quality, physicochemical, microbiological, biological analysis, pollution, population. 


\section{INTRODUCCIÓN}

La quebrada El Horno uno de los tributarios del Rio Abajo cuenta con una extensión de $4.72 \mathrm{Km} 2$, este tributario sirve como fuente de abastecimiento de agua (aseo personal y uso doméstico) para los pobladores de la colonia Nueva Esperanza, que se ubica en la ciudad de Danlí, departamento de El Paraíso (ver Figura 1). Dicha colonia entre 2008-2009 participo en una licitación para poder formar parte del proyecto piloto barrio-ciudad (El Heraldo, 2014), el cual beneficio de cerca de 17 mil habitantes con proyectos tales como alcantarillados sanitarios y pavimentación.

El agua dulce es un recurso limitado y su calidad está bajo presión constante. Preservar la calidad del agua dulce es importante para el abastecimiento de agua potable, la producción de alimentos y el uso de aguas recreativas (OMS., 2009).

Por otro lado, el agua tiene dos dimensiones que están interrelacionadas: calidad y cantidad. El concepto específico sobre la calidad del agua se ha ido modificando constantemente da un enfoque estrictamente fisicoquímico a otro que integra los componentes del ecosistema. Los sistemas dulceacuícolas tienen una importancia fundamental, de ahí la necesidad de en los últimos años la necesidad de regular el estado de conservación y afianzar así una adecuada calidad del agua, fomentando la realización de estudios integrales en los que también se incluye el análisis de distintos grupos de seres vivos (Castellon, 2013).

Las comunidades de seres vivos son el reflejo de las condiciones fisicoquímicas que existen en el ecosistema y a su vez proporcionan información sobre el estado de las aguas en un periodo de tiempo previo al momento de cualquier muestreo, debido a que son sensibles a distintas alteraciones que puede sufrir el medio acuático. Los macroinvertebrados acuáticos, al igual que las diatomeas, macrófitas y peces, son parte de los grupos más frecuentemente utilizados en los últimos años en estudios de la calidad del agua (Rodriguez \& Gentil, 2012).

Más concretamente, se consideran macroinvertebrados acuáticos a todos aquellos organismos invertebrados que habitan en algún momento de su ciclo vital en los cuerpos de agua, y que pueden ser retenidos por mallas con luz de entre 200 y $500 \mu \mathrm{m}$. Esto incluye una gran cantidad de especies de distintos grupos como los anélidos, moluscos, platelmintos, nematodos y artrópodos (principalmente insectos) (Ladrera et al., 2013). 
Figura 1. Ubicación espacial de la investigación

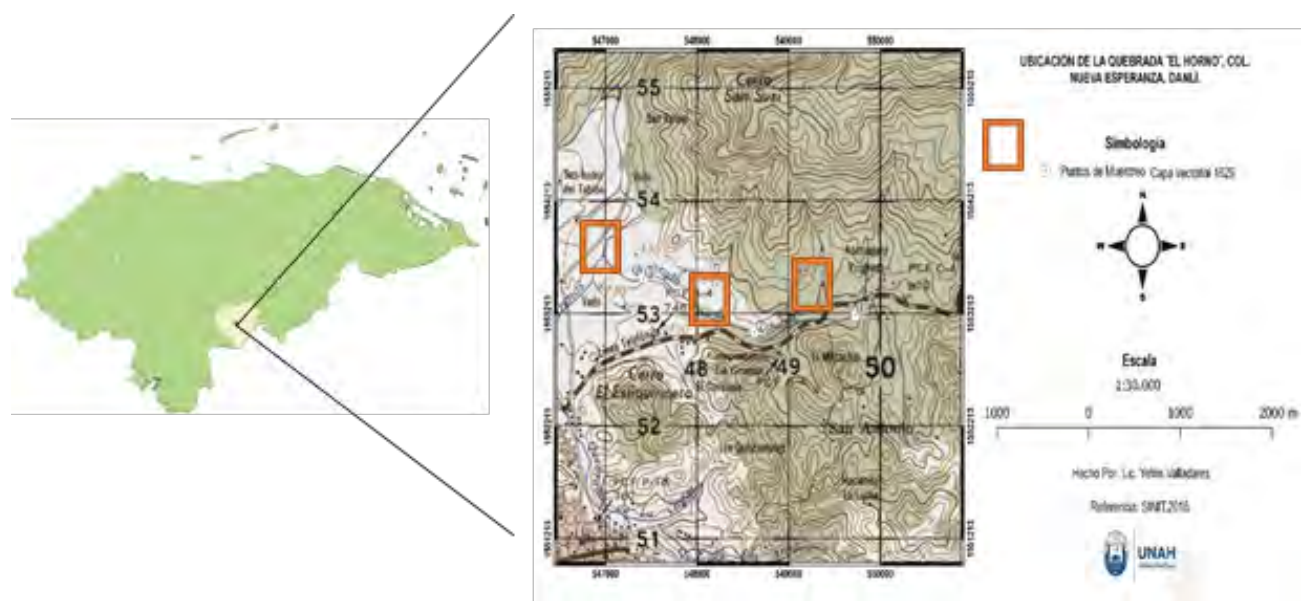

La presencia de sustancias químicas disueltas e insolubles en el agua puede ser de origen natural 0 antropogénico. Se ha de tomar en cuenta también, debido a la amplia gama de contaminantes, los diferentes niveles de contaminación, así como la cinética química de las sustancias, elementos, materia orgánica y microorganismos que se incorporan en el cuerpo de agua, ya que es indispensable para determinar características físicas y químicas (Barrenechea, 2004).

El control de los parámetros fisicoquímicos y microbiológicos es importante tanto en los sistemas de potabilización como de depuración del agua. Sin embargo, en los lugares donde el agua es consumida por el hombre o es reutilizada, el factor de riesgo más importante está asociado con la exposición a agentes microbiológicos que incluyen bacterias patógenas, helmintos, protozoos y virus entéricos (Asano, 1998).

\section{METODOLOGÍA}

Se establecieron los puntos de muestreo a lo largo de la quebrada El Horno (Imagen 1) donde se buscaron tres localidades equidistantes en relación con la extensión de cuenca: 
Tabla 1. Puntos de muestreos en la quebrada El Horno

\begin{tabular}{|l|l|c|c|}
\hline \multicolumn{1}{|c|}{ Cuenca } & Nombre & $\begin{array}{c}\text { Coordenadas X } \\
\text { (UTM) }\end{array}$ & $\begin{array}{c}\text { Coordenada Y } \\
\text { (UTM) }\end{array}$ \\
\hline Cuenca alta & El Túnel & 549730.03 & 1553205.93 \\
\hline Cuenca media & Puente & 548625.73 & 1553054.72 \\
\hline Cuenca baja & Final & 547416.03 & 1553535.84 \\
\hline
\end{tabular}

Fuente: datos propios.

Se identificaron las fuentes de contaminación del efluente a partir de las visitas de campo y encuestas levantadas por los alumnos de la Carrera de Enfermería de la UNAH-TEC Danlí, las cuales constaban de preguntas acerca de la utilidad que los habitantes de la zona le daban al agua de la quebrada y también si han padecido enfermedades gastrointestinales y alergias. La colecta de macroinvertebrados (ver Figura 2a), así como la toma de muestras de los parámetros fisicoquímicos y microbiológicos (ver Figura 2b), se realizó una vez al mes durante tres meses (septiembre - noviembre del 2016).

Figura 2. (a) Colecta de macroinvertebrados, (b) toma de muestra química y microbiológica.

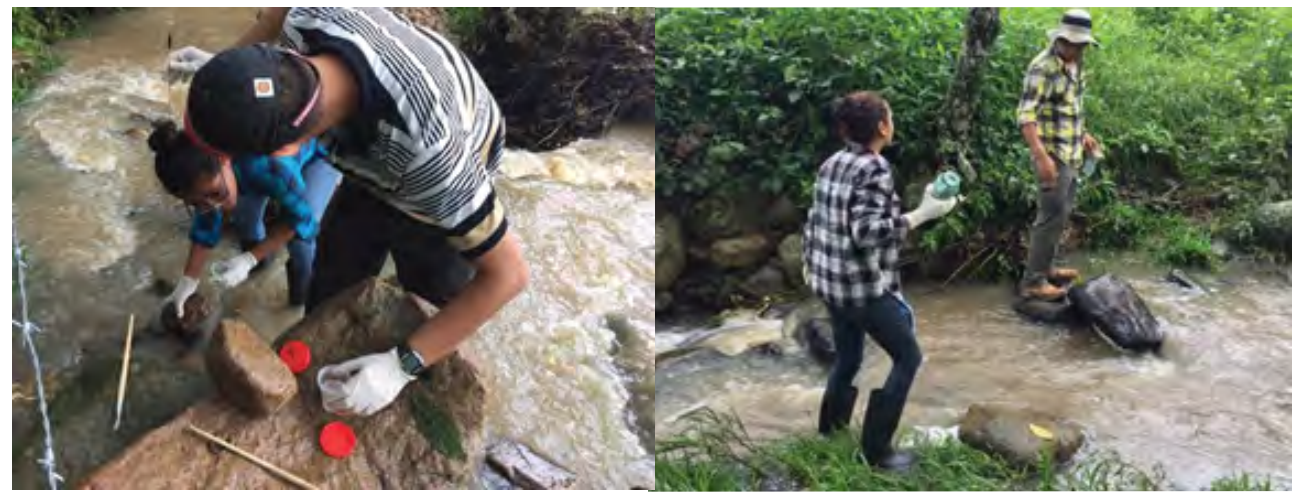

Fuente: datos propios.

\section{Muestreo fisicoquímico}

Se tomaron muestra simples en botellones de un galón de capacidad para determinar parámetros fisicoquímicos tales como alcalinidad, aluminio, cadmio, calcio, cianuro, cloro, cobalto, cobre, color, conductividad (a partir de conductímetro), cromo, detergentes, DQO, fluor, formaldehido, fosfatos, hierro, magnesio, nitratos, oxígeno disuelto (a partir de oxinómetro), pH (a partir de pHmetro), plomo, potasio, salinidad (a partir 
de salinómetro), solidos suspendidos, sulfuros, temperatura del agua (a partir de termómetro) y zinc. Estos parámetros fueron cuantificados a través de espectrofotometría en el laboratorio de química de la UNAH-TEC Danlí. Estos parámetros fueron comparados y normados bajo la Propuesta Nacional de Norma para el Agua en Honduras (Secretaria de Salud/CAPRE, 2001). Los parámetros fueron tomados con el uso de espectroscopio. Parámetros físicos fueron tomados con sonda multiparamétrica.

\section{Muestreo microbiológico}

Las muestras microbiológicas fueron tomadas en bolsas bacteriológicas de $100 \mathrm{ml}$. Luego estas se transportaron embaladas con hielo para evitar el crecimiento de bacterias luego de la colecta. Fueron analizadas en el laboratorio de microbiología de la UNAH-TEC Danlí. La cuantificación de bacterias de cada análisis fue comparado y normado bajo la Propuesta Nacional de Norma para el Agua en Honduras (Secretaria de Salud/CAPRE, 2001).

\section{Muestreo biológico}

Para la colecta de macroinvertebrados (Alba \& Sanchez, 1988) se utilizaron botes de plástico estériles. Con ayuda de pinzas y pinceles, se colectaron los organismos encontrados bajo las rocas y sobre la película de agua de la quebrada. Para la preservación de los macroinvertebrados, se utilizó alcohol al 95\%. Los organismos fueron identificados en el laboratorio de biología de la UNAH-TEC Danlí con la ayuda de un estereoscopio (4x) y las claves taxonómicas de Merritt et al. (2008) y Roldan (1988). Para determinar la calidad del agua a partir de organismos biológicos (Tabla 2) se utilizaron los índices BMWP-CR (La Gaceta , 2007) e IBF-SV2010 (Sermeño et al., 2010)

Se extrapolaron datos de cada muestreo usado Microsoft Excel® para la generación de tablas y gráficas.

\section{RESULTADOS}

A continuación, se presenta los diferentes resultados obtenidos para los análisis fisicoquímicos, microbiológicos y biológicos durante la investigación realizada en la quebrada El Horno. 
Tabla 2. Índices biológicos usados en la investigación

\begin{tabular}{|l|l|l|}
\hline \multicolumn{2}{|c|}{ BMWP-CR } & Categorías \\
\hline \multicolumn{1}{|c|}{ Rangos } & \multicolumn{1}{|c|}{ Nivel de Contaminación } & \\
\hline$>120$ & Agua de calidad excelente & \\
\hline $101-120$ & Agua de calidad buena, no contaminadas & \\
\hline $61-100$ & Agua de calidad regular, contaminación moderada & \\
\hline $36-60$ & Agua de calidad mala, contaminada & \\
\hline $16-35$ & Agua de calidad mala, muy contaminada & \\
\hline$<15$ & Agua de calidad mala, extremadamente contaminada & \\
\hline \multicolumn{3}{|c|}{ IBF-SV2010 } \\
\hline $0.00-3.75$ & Excelente, contaminación orgánica improbable & \\
\hline $3.76-4.25$ & Muy buena, contaminación orgánica leve posible & \\
\hline $4.26-5.00$ & Buena, alguna contaminación orgánica probable & \\
\hline $5.01-5.75$ & Regular, contaminación orgánica sustancial & \\
\hline $5.76-6.50$ & Regular pobre, contaminación sustancial probable & \\
\hline $6.51-7.25$ & Pobre, contaminación muy sustancial & \\
\hline $7.26-10.00$ & Muy pobre, contaminación orgánica severa & \\
\hline
\end{tabular}

Fuente: Modificado de La Gaceta (2007) para BMWP-CR y de Sermeño et al. (2010) para IBF-SV2010

\section{Análisis Fisicoquímico del agua:}

\section{a. Interpretación de cada uno de los puntos de muestreos:}

Entre los parámetros que no cumplen con la normativa de calidad de agua en Honduras y la norma CAPRE $(0.20 \mathrm{mg} / \mathrm{l})$ podemos mencionar el aluminio, el cual se encuentra en concentraciones de $0.317 \mathrm{mg} / \mathrm{l}$ (Tabla 3 ) como valor máximo y una media de $0.219 \mathrm{mg} / \mathrm{l}$ en el punto 1 , valor de $0.313 \mathrm{mg} / \mathrm{l}$ como máximo y de $0.227 \mathrm{mg} / \mathrm{l}$ como valor medio en el punto 2 y en el caso del punto 3 se encuentra un valor máximo de $0.291 \mathrm{mg} / \mathrm{l}$ y una media de $0.220 \mathrm{mg} / \mathrm{l}$.

El color es una característica organoléptica del agua. La normativa de calidad del agua indica que el valor del color del agua para cualquier actividad humana no debe exceder 15 UC. Para el punto 1 el valor máximo es de 27 UC (Tabla 3) y una media de 20.666 UC. En el punto 2 el valor máximo encontrado es 57 UC y una media de 39.333 UC. El punto 3 tiene un valor máximo de 43 UC y un valor medio de 31.666 UC. 
Tabla 3. Análisis fisicoquímicos de la quebrada El Horno

\begin{tabular}{|c|c|c|c|c|c|c|c|c|c|c|c|}
\hline \multicolumn{12}{|c|}{ Muestreo de parámetros fisicoquímicos de la quebrada El Horno } \\
\hline \multirow[b]{3}{*}{ Análisis } & \multirow[b]{3}{*}{ Unidad } & \multirow{3}{*}{$\begin{array}{c}\text { Normativa } \\
\text { de calidad } \\
\text { de agua }\end{array}$} & \multirow{2}{*}{\multicolumn{3}{|c|}{$\begin{array}{c}\text { Punto } 1 \text { El Túnel } \\
\text { muestreo } \\
\end{array}$}} & \multirow{2}{*}{\multicolumn{3}{|c|}{$\begin{array}{c}\text { Punto } 2 \text { Puente } \\
\text { muestreo }\end{array}$}} & \multirow{2}{*}{\multicolumn{3}{|c|}{$\begin{array}{c}\text { Punto } 3 \text { Final } \\
\text { muestreo }\end{array}$}} \\
\hline & & & & & & & & & & & \\
\hline & & & 1 & 2 & 3 & 1 & 2 & 3 & 1 & 2 & 3 \\
\hline alcalinidad & $\mathrm{mg} / \mathrm{l}$ & $300.00^{\mathrm{a}}$ & 125 & 93.5 & 141 & 84 & 72.4 & 41.8 & 67.3 & 56.1 & 119 \\
\hline aluminio & $\mathrm{mg} / \mathrm{l}$ & 0.20 & 0.317 & 0.19 & 0.15 & 0.313 & 0.21 & 0.16 & 0.292 & 0.22 & 0.15 \\
\hline cadmio & $\mathrm{mg} / \mathrm{l}$ & 0.003 & 0 & 0 & 0 & 0 & 0 & 0 & 0 & 0 & 0 \\
\hline calcio & $\mathrm{mg} / \mathrm{l}$ & SN & 6.34 & 6.61 & 8.4 & 6.39 & 6.62 & 5.7 & 6.36 & 7.18 & 6.32 \\
\hline cianuro & $\mathrm{mg} / \mathrm{l}$ & 0.07 & 0.005 & 0.001 & 0.007 & 0.003 & 0 & 0.005 & 0.005 & 0.001 & 0.006 \\
\hline cloruro & $\mathrm{mg} / \mathrm{l}$ & 250.00 & 0.09 & 0.4 & 0.06 & 0.06 & 0.32 & 0.1 & 0.09 & 0.42 & 0.47 \\
\hline cobalto & $\mathrm{mg} / \mathrm{l}$ & $0.05^{a}$ & 0.01 & 0.05 & 0.01 & 0 & 0.15 & 0.01 & 0.01 & 0.11 & 0.03 \\
\hline cobre & $\mathrm{mg} / \mathrm{l}$ & 2.00 & 0.02 & 0.01 & 0.03 & 0.03 & 0.11 & 0.02 & 0.03 & 0 & 0.02 \\
\hline color & UC & 15.00 & 18 & 27 & 17 & 11 & 50 & 57 & 13 & 43 & 39 \\
\hline conductividad & $\mu \mathrm{s} / \mathrm{cm}$ & 1000.00 & 212 & 104 & 308 & 234 & 176 & 398 & 244 & 152 & 445 \\
\hline cromo & $\mathrm{mg} / \mathrm{l}$ & 0.05 & 0.01 & 0.001 & 0.021 & 0.009 & 0.001 & 0.004 & 0.005 & 0.007 & 0 \\
\hline detergentes & $\mathrm{mg} / \mathrm{l}$ & SN & 0.004 & 0.003 & 0.003 & 0.005 & 0.003 & 0 & 0.014 & 0.032 & 0.017 \\
\hline DQO & $\mathrm{mg} / \mathrm{l}$ & 10.00 & 937 & 330 & 281 & 680 & 234 & 422 & 0 & 331 & 84 \\
\hline fluoruro & $\mathrm{mg} / \mathrm{l}$ & 1.5 & 0 & 0 & 0 & 0 & 0 & 0 & 0 & 0 & 0 \\
\hline formaldehido & $\mu \mathrm{g} / \mathrm{l}$ & SN & 106 & 0 & 140 & 140 & 68 & 153 & 170 & 12 & 219 \\
\hline fosfatos & $\mathrm{mg} / \mathrm{l}$ & 0.10 & 0.11 & 0.22 & 0.29 & 0.06 & 0.22 & 0.11 & 0.08 & 0.13 & 0.17 \\
\hline hierro & $\mathrm{mg} / \mathrm{l}$ & 0.30 & 0.58 & 0.81 & 0.44 & 0.27 & 0.77 & 0.44 & 0.24 & 0.76 & 0.2 \\
\hline magnesio & $\mathrm{mg} / \mathrm{l}$ & SN & 2.87 & 3.13 & 3.95 & 3 & 3.1 & 2.65 & 2.96 & 3.37 & 2.98 \\
\hline nitratos & $\mathrm{mg} / \mathrm{l}$ & 50.00 & 0.4 & 0.2 & 2 & 0.1 & 0.2 & 3 & 0.2 & 0.2 & 3 \\
\hline OD & $\mathrm{mg} / \mathrm{l}$ & 5.00 & 9.6 & 8.3 & 9.3 & 9.2 & 10.9 & 10.3 & 8.8 & 7.5 & 10.7 \\
\hline $\mathrm{Ph}$ & & $6.5-8.5$ & 7.88 & 6 & 7.54 & 7.99 & 6.77 & 8.3 & 7.85 & 7.02 & 8.56 \\
\hline plomo & $\mu g / l$ & 0.01 & 0 & 0 & 0 & 0 & 0 & 0 & 0 & 0 & 0 \\
\hline potasio & $\mathrm{mg} / \mathrm{l}$ & 10.00 & 2 & 0.9 & 0.8 & 0.5 & 0.5 & 0.9 & 1.8 & 0.5 & 2.1 \\
\hline salinidad & $\mathrm{mg} / \mathrm{l}$ & SN & 0.32 & 0.21 & 0.44 & 0.11 & 0.06 & 0.32 & 0.11 & 0.08 & 1.54 \\
\hline SS & $\mathrm{mg} / \mathrm{l}$ & $80.00^{a}$ & 3 & 64 & 2 & 3 & 93 & 15 & 5 & 86 & 5 \\
\hline sulfuros & $\mathrm{mg} / \mathrm{l}$ & 250.00 & 3 & 24 & 4 & 4 & 38 & 20 & 4 & 36 & 12 \\
\hline temp agua & ${ }^{\circ} \mathrm{C}$ & SN & 22 & 22 & 23 & 25 & 24 & 25 & 28 & 26 & 28 \\
\hline zinc & $\mathrm{mg} / \mathrm{l}$ & 3.00 & 0.3 & 0.1 & 0.3 & 0.02 & 0.03 & 0.02 & 0.03 & 0.03 & 0 \\
\hline
\end{tabular}

Fuente: datos propios. *El término SN hace referencia a que el parámetro no se encuentra normado, cada uno de los datos fueron comparados con la Normativa de Calidad de Agua (Secretaria de Salud/CAPRE, 2001), (a) normado con parámetros utilizados para uso agrícola, los demás están bajo el apéndice agua de uso de abastecimiento para la población (Categoría A). 
La demanda química de oxígeno (DQO) es un parámetro de suma importancia que determina como se da el consumo de oxígeno por los cuerpos reductores presentes en el agua sin la intervención de los organismos vivos. El máximo permisible por normativa de la calidad de agua en Honduras es de $20 \mathrm{mg} / \mathrm{l}$ para el uso humano y de $50 \mathrm{mg} / \mathrm{l}$ en el caso de la preservación de la flora y fauna. El punto 1 tiene un valor máximo de $937 \mathrm{mg} / \mathrm{l}$ (Tabla 3) y una media de $516 \mathrm{mg} / \mathrm{l}$. Para el punto 2 el valor máximo es de $680 \mathrm{mg} / \mathrm{l}$ y una media $445.333 \mathrm{mg} / \mathrm{l}$. Para el punto 3 de muestreo el valor máximo es de $331 \mathrm{mg} / \mathrm{l}$ y una media $138.333 \mathrm{mg} / \mathrm{l}$.

Para las concentraciones máximas permitidas de fosfatos en el agua, según normativa hondureña es de $0.10 \mathrm{mg} / \mathrm{l}$ la mayor concentración de fosfatos en el punto 1 es de $0.290 \mathrm{mg} / \mathrm{l}$ (Tabla 3) y una media de $0.201 \mathrm{mg} / \mathrm{l}$. El punto 2 tiene una concentración máxima de $0.220 \mathrm{mg} / \mathrm{l}$ y una media $0.136 \mathrm{mg} / \mathrm{l}$. En el caso del punto 3, el valor máximo encontrado en el análisis es de $0.170 \mathrm{mg} / \mathrm{l}$ y una media de $0.126 \mathrm{mg} / \mathrm{l}$.

El Hierro, elemento el cual tampoco cumple con la normativa de calidad de agua en donde el valor máximo es de $0.30 \mathrm{mg} /$. Para el punto 1, el valor máximo encontrado es de $0.880 \mathrm{mg} / \mathrm{L}$ (Tabla 3) y un valor medio de $0.633 \mathrm{mg} / \mathrm{l}$. En el punto 2 de muestreo, máximo es de $0.770 \mathrm{mg} / \mathrm{l}$ y una media $0.493 \mathrm{mg} / \mathrm{l}$. En el punto 3 , el valor máximo es de $0.760 \mathrm{mg} / \mathrm{l}$ y una media de $0.413 \mathrm{mg} / \mathrm{l}$.

\section{b. Análisis de los puntos de muestreo:}

Como ya se pudo constatar en el apartado anterior y en la Tabla 3, existe contaminación química de la quebrada El Horno en los tres puntos de muestreo.

\section{Grafica 1. Parámetros que superan la normativa de calidad de agua.}

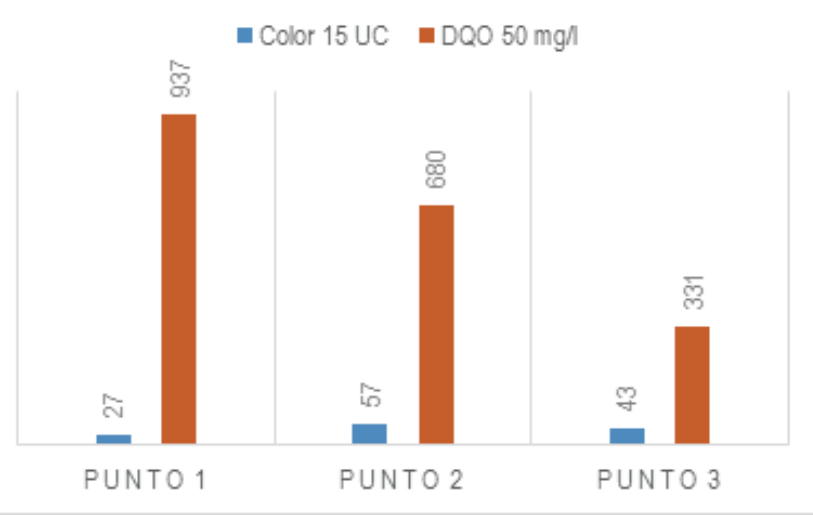

Fuente: datos propios 
El nivel más alto de contaminación se encuentra en el punto 1. Uno de los parámetros con valores más altos y que genera mayor preocupación en el Punto 1 es la DQO (ver Grafica 1). Esto es indicativo de una gran cantidad de agentes reductores que está consumiendo el oxígeno de la quebrada. Sin embargo, al medir la cantidad de oxígeno con la sonda multiparamétrica, los niveles de oxígeno se encontraban dentro de lo permitido por la norma. El punto 2 presenta los valores más altos en las unidades de color (ver Grafica 1). En este caso cabe mencionar que éste se encuentra en el centro de la colonia Nueva Esperanza en donde la actividad antropogénica tiene repercusiones sobre las unidades de color.

\section{Gráfica 2. Elementos que exceden la normativa de calidad de agua.}

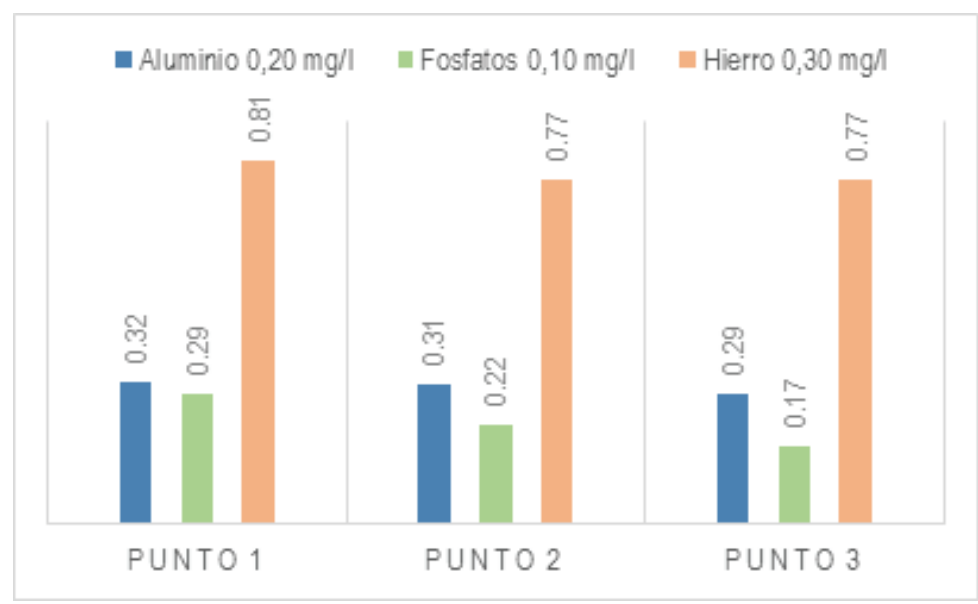

Fuente: datos propios.

Si se toma en cuenta el conjunto de datos químicos analizados, la parte alta de la cuenca (punto 1) tiene el mayor grado de contaminación. En sus alrededores se encuentra una purificadora de agua y una amplia zona ganadera la cual se ha extendido en los últimos años. Estas dos actividades pueden estar contaminando la quebrada en gran parte. Los altos niveles de aluminio presentes en los tres muestreos realizados puede ser efecto de los productos usados en la purificación del agua, ya que es un elemento importante en la generación de fóculos.

\section{Análisis microbiológico del agua:}

A nivel mundial, uno de los principales marcadores microbiológicos de la contaminación del agua han sido los microorganismos clasificados dentro de los coliformes. A continuación, se presentan los análisis microbiológicos que se realizaron durante el periodo de investigación. 
Tabla 4. Análisis microbiológico de la quebrada El Horno

\begin{tabular}{|c|c|c|c|c|c|c|c|c|c|c|c|}
\hline \multicolumn{12}{|c|}{ Muestreo de parámetros microbiológicos de la quebrada El Horno } \\
\hline \multirow{3}{*}{ Análisis } & \multirow{3}{*}{ Unidad } & \multirow{3}{*}{$\begin{array}{c}\text { Normativa } \\
\text { de Calidad } \\
\text { de Agua }\end{array}$} & \multirow{2}{*}{\multicolumn{3}{|c|}{$\begin{array}{c}\text { Punto } 1 \text { El Túnel } \\
\text { Muestreo }\end{array}$}} & \multirow{2}{*}{\multicolumn{3}{|c|}{$\begin{array}{c}\text { Punto } 2 \text { Puente } \\
\text { Muestreo }\end{array}$}} & \multirow{2}{*}{\multicolumn{3}{|c|}{$\begin{array}{c}\text { Punto } 3 \text { Final } \\
\text { Muestreo }\end{array}$}} \\
\hline & & & & & & & & & & & \\
\hline & & & $\overline{1}$ & 2 & 3 & 1 & 2 & 3 & 1 & 2 & 3 \\
\hline $\begin{array}{l}\text { Coliformes } \\
\text { totales }\end{array}$ & $\begin{array}{l}\text { UFC/100 } \\
\mathrm{mL}\end{array}$ & 10 & Incon & Incon & Incon & Incon & Incon & Incon & Incon & Incon & Incon \\
\hline $\begin{array}{l}\text { Coliformes } \\
\text { fecales }\end{array}$ & $\begin{array}{l}\text { UFC/100 } \\
\mathrm{mL}\end{array}$ & 0 & Incon & Incon & Incon & Incon & Incon & Incon & Incon & Incon & Incon \\
\hline
\end{tabular}

Fuente: datos propios. Normados bajo la normativa de ley para la calidad del agua (Secretaria de Salud/CAPRE, 2001). *El término Incon hace referencia a incontable.

\section{a. Interpretación de cada uno de los puntos de muestreos:}

La normativa de calidad de agua en Honduras /CAPRE, en cuanto al tema microbiológico del agua, ha sido muy estricta. Se ha establecido la cantidad 10 UFC/100 mL (coliformes totales) y 0 UFC/100 mL (coliformes fecales) para agua de abastecimiento no entubada, el cual es el caso de la quebrada El Horno. En cada uno de los muestreos, la cuantificación es de incontables UFC/100 mL. Esto quiere decir que, aunque el método utilizado es cuantitativo, la cantidad de colonias es tan grande que no se pueden cuantificar con el ojo humano (Tabla 4).

\section{b. Análisis de los puntos de muestreo:}

Los resultados en cuanto a los análisis microbiológicos del agua son aún más contundentes para asegurar la alta contaminación que tiene la quebrada El Horno.

A lo largo de la quebrada, se observó la urbanización de la zona ripiaría y la actividad ganadera, sin dejar por un lado las industrias que se encuentran en la parte alta. En la parte media podemos encontrar que algunos de los colectores de aguas negras se encuentran en el cauce de la quebrada. Además, muchos de las casas en este tramo no cuentan con sistema de aguas negras, lo cual ha hecho que los habitantes, en su mayoría, hagan sus necesidades en las riveras de la quebrada. Por otro lado, tuberías ubicadas clandestinamente llevan heces fecales al caudal de la quebrada, causando la contaminación por patógenos microbiológicos, en este caso, los coliformes fecales. Esto se extiende hasta la zona baja de la quebrada donde el sistema de aguas negras es inexistente y en donde se concentra la mayor cantidad de los remanentes de contaminación por coliformes. 


\section{Análisis biológico del agua:}

Los análisis utilizando bioindicadores son cada vez más comunes, ya que éstos son una manera económica de saber el estado de conservación y calidad de un sitio de estudio. Esta investigación centra un apartado en los macroinvertebrados, un grupo heterogéneo de insectos que colonizan las aguas de todos los continentes.

\section{a. Interpretación de cada muestreo:}

Al igual que en los análisis anteriores, la parte biológica se dividió en puntos, lo cual permitió analizar por separado el estado biológico de la quebrada El Horno. Se cuantifico un total de 1,401 individuos distribuidos en un total de 46 familias. Además, entre las familias más representativas se encuentran: Simuliidae (560 individuos), Chironomidae (373 individuos) y Beatidae (221 individuos).

Si observamos la Tabla 5, en la parte inferior podemos observar que el BMWP-CR da un total de 86. En vista que este valor cae en el rango de 61-100 de la Tabla 2, se determinó que el agua es de calidad regular y contaminación moderada. Por otro lado, el valor de IBF-SV2010 para el primer muestreo es de 6.13, y como este valor cae en el rango de 5.76 a 6.50 en la Tabla 2, se determinó que el agua es de calidad pobre a regular y con una contaminación sustancial probable.

Para el segundo muestreo, la Tabla 6 indica un valor de BMWP-CR de 99 en vista que este valor cae en el rango de 61 a 100 de la Tabla 2, se determinó que el agua es de pobre calidad y con contaminación moderada. La Tabla 6 muestra un valor de IBF-SV2010 de 5.95 y debido a que cae en el rango de 5.76-6.50 de la Tabla 2, se determinó que el agua es regular pobre, contaminación sustancial probable

Para el último muestreo, el BMWP-CR tiene un valor de 82 (Tabla 7) y se encuentra en el rango de 61 a 100 de la Tabla 2 (agua de clidad pobre y contaminación moderada). El IBF-SV2010 se encuentra en 6.55 y se encuentra en el rango de 6.51 a 7.25 de la Tabla 2, por lo que se determinó que el agua es pobre y con una contaminación muy sustancial probable. 
Tabla 5. Primer muestreo biológico realizado.

\begin{tabular}{|c|c|c|c|c|c|c|c|c|c|c|c|c|c|c|c|}
\hline \multicolumn{16}{|c|}{ Muestreo de parámetros biológicos de la Quebrada El Horno (septiembre de 2016) } \\
\hline \multirow[b]{3}{*}{ 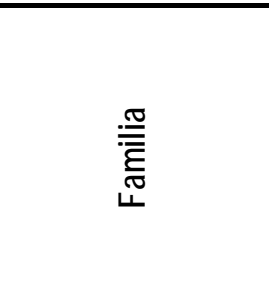 } & \multicolumn{5}{|c|}{ Punto 1 El Túnel } & \multicolumn{5}{|c|}{ Punto 2 Puente } & \multicolumn{5}{|c|}{ Punto 3 Final } \\
\hline & \multirow[b]{2}{*}{ 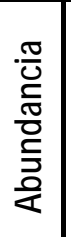 } & \multirow[b]{2}{*}{ 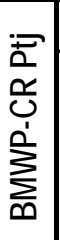 } & \multicolumn{3}{|c|}{ IBF-SV2010 } & \multirow[b]{2}{*}{ 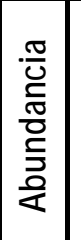 } & \multirow[b]{2}{*}{ 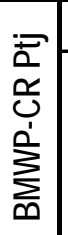 } & \multicolumn{3}{|c|}{ IBF-SV2010 } & \multirow[b]{2}{*}{ 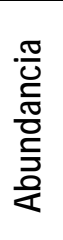 } & \multirow[b]{2}{*}{ 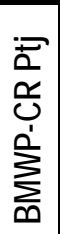 } & \multicolumn{3}{|c|}{ IBF-SV2010 } \\
\hline & & & 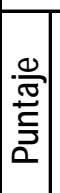 & 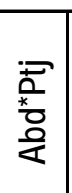 & 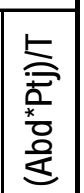 & & & 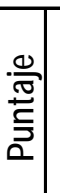 & 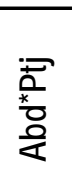 & $\begin{array}{l}\text { 上 } \\
\text { 竞 } \\
\frac{*}{0} \\
\frac{0}{\leq}\end{array}$ & & & 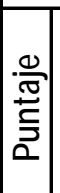 & 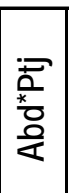 & 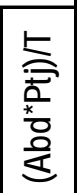 \\
\hline Simuliidae & 129 & 4 & 6 & 774 & 4.32 & 80 & 4 & 6 & 480 & 2.33 & 9 & 4 & 6 & 54 & 0.84 \\
\hline Ceratopogonidae & 7 & 4 & 8 & 56 & 0.31 & - & - & - & - & - & - & - & - & - & - \\
\hline Empididae & 1 & 4 & 6 & 6 & 0.03 & - & - & - & - & - & - & - & - & - & - \\
\hline Chironomidae & 12 & 2 & 8 & 96 & 0.54 & 18 & 2 & 8 & 144 & 0.70 & 19 & 2 & 8 & 152 & 2.38 \\
\hline Potamanthidae & 2 & - & - & - & - & 3 & - & - & - & - & 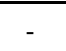 & - & - & - & - \\
\hline Culicidae & 2 & 2 & 10 & 20 & 0.11 & - & - & - & - & - & - & - & - & - & - \\
\hline Baetidae & 12 & 5 & 6 & 72 & 0.40 & 81 & 5 & 6 & 486 & 2.36 & 26 & 5 & 6 & 156 & 2.44 \\
\hline Ecnomidae & 1 & 10 & - & - & - & - & - & - & - & - & - & - & - & - & - \\
\hline Glossosomatid & 1 & 10 & 1 & 1 & 0.01 & - & - & - & - & - & - & - & - & - & - \\
\hline Haliplidae & 1 & 4 & - & - & - & - & - & - & - & - & - & - & - & - & - \\
\hline Siphlonuridae & 2 & - & - & - & - & - & - & - & - & - & - & - & - & - & - \\
\hline Scirtidae & 1 & 4 & 6 & 6 & 0.03 & - & - & - & - & - & - & - & - & - & - \\
\hline Dytiscidae & 1 & 4 & 7 & 7 & 0.04 & - & - & - & - & - & - & - & - & - & - \\
\hline Hydroptilidae & 2 & 6 & 4 & 8 & 0.04 & - & - & - & - & - & 1 & 6 & 4 & 4 & 0.06 \\
\hline Brachycentridae & 3 & - & - & - & - & 3 & - & - & - & - & 2 & - & - & - & - \\
\hline Nepidae & 1 & 4 & 5 & 5 & 0.03 & - & - & - & - & - & - & - & - & - & - \\
\hline Corydalidae & 1 & 6 & 7 & 7 & 0.04 & 1 & 6 & 7 & 7 & 0.0 & 1 & 6 & 7 & 7 & 0.11 \\
\hline Veliidae & - & - & - & - & - & 4 & - & 5 & 20 & 0.10 & - & - & - & - & - \\
\hline Caenidae & - & - & - & - & - & 7 & 4 & 7 & 49 & 0.24 & 4 & 4 & 7 & 28 & 0.44 \\
\hline Platycnemididae & - & - & - & - & - & 4 & - & 1 & 4 & 0.02 & - & - & - & - & - \\
\hline Planariidae & - & - & - & - & - & 2 & - & 5 & 10 & 0.05 & - & - & - & - & - \\
\hline Libellulidae & - & - & - & - & - & 1 & 6 & 7 & 7 & 0.03 & - & - & - & - & - \\
\hline Psephenidae & - & - & - & - & - & 2 & 7 & 4 & 8 & 0.04 & - & - & - & - & - \\
\hline Syrphidae & - & - & - & - & - & - & - & - & - & - & 2 & 1 & 10 & 20 & 0.31 \\
\hline Total (T) & 179 & 69 & & & 5.91 & 206 & 34 & & & 5.90 & 64 & 28 & & & 6.58 \\
\hline BMWPICR total & & & & & & & & 86 & & & & & & & \\
\hline IBF/SV2010 total & & & & & & & & 0.1 & & & & & & & \\
\hline Abd total & & & & & & & & & & & & & & & \\
\hline
\end{tabular}

Fuentes: datos propios y comparados con La Gaceta (2007) y Sermeño et al. (2010). Nota: se observan abreviaturas para abundancia (Abj), puntaje (Ptj). Las casillas color anaranjado corresponden al resultado del índice por punto de muestreo. 
Tabla 6. Segundo muestreo biológico realizado.

\begin{tabular}{|c|c|c|c|c|c|c|c|c|c|c|c|c|c|c|c|}
\hline \multicolumn{16}{|c|}{ Muestreo de parámetros biológicos de la Quebrada El Horno (octubre de 2016) } \\
\hline \multirow[b]{3}{*}{ 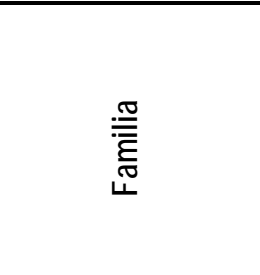 } & \multicolumn{5}{|c|}{ Punto 1 El Túnel } & \multicolumn{5}{|c|}{ Punto 2 Puente } & \multicolumn{5}{|c|}{ Punto 3 Final } \\
\hline & \multirow[b]{2}{*}{ 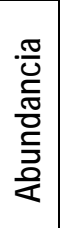 } & \multirow[b]{2}{*}{ 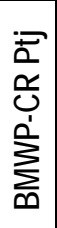 } & \multicolumn{3}{|c|}{ IBF-SV2010 } & \multirow[b]{2}{*}{ 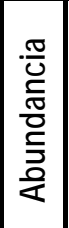 } & \multirow[b]{2}{*}{ 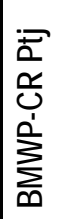 } & \multicolumn{3}{|c|}{ IBF-SV2010 } & \multirow[b]{2}{*}{$\begin{array}{l}\frac{\pi}{0} \\
\frac{c}{0} \\
\frac{\pi}{0} \\
\frac{C}{3} \\
\frac{0}{1}\end{array}$} & \multirow[b]{2}{*}{ 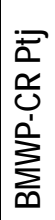 } & \multicolumn{3}{|c|}{ IBF-SV2010 } \\
\hline & & & 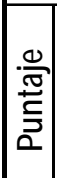 & 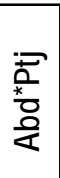 & 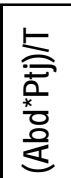 & & & 产 & 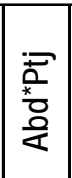 & 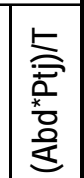 & & & 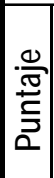 & 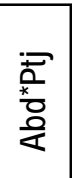 & 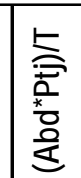 \\
\hline Simuliidae & 14 & 4 & 6 & 84 & 2.47 & 121 & 4 & 6 & 726 & 2.69 & 7 & 4 & 6 & 42 & 0.39 \\
\hline Ceratopogonidae & 1 & 4 & 8 & 8 & 0.24 & - & - & - & - & - & - & - & - & - & - \\
\hline Chironomidae & 3 & 2 & 8 & 24 & 0.71 & 30 & 2 & 8 & 240 & 0.89 & 52 & 2 & 8 & 416 & 3.82 \\
\hline Potamanthidae & 1 & - & - & & - & 19 & - & - & - & - & 1 & - & - & - & - \\
\hline Baetidae & 3 & 5 & 6 & 18 & 0.53 & 35 & 5 & 6 & 210 & 0.78 & 29 & 5 & 6 & 174 & 1.60 \\
\hline Glossosomatidae & 1 & 10 & 1 & 1 & 0.03 & - & - & - & - & - & - & - & - & - & - \\
\hline Scirtidae & 1 & 4 & 6 & 6 & 0.18 & - & - & - & - & - & - & - & - & - & - \\
\hline Dytiscidae & 1 & 4 & 7 & 7 & 0.21 & - & - & - & - & - & - & - & - & - & - \\
\hline Veliidae & 1 & - & 5 & 5 & 0.15 & 2 & 0 & 5 & 10 & 0.04 & - & - & - & - & - \\
\hline Caenidae & 2 & 4 & 7 & 14 & 0.41 & 5 & 4 & 7 & 35 & 0.13 & 11 & 4 & 7 & 77 & 0.71 \\
\hline Syrphidae & 1 & 1 & 10 & 10 & 0.29 & - & - & - & - & - & - & - & - & - & - \\
\hline Helicopsychidae & 1 & 5 & 5 & 5 & 0.15 & - & - & - & - & - & - & - & - & - & - \\
\hline Gerridae & 1 & - & 6 & 6 & 0.18 & 5 & - & 6 & 30 & 0.11 & - & - & - & - & - \\
\hline Tipulidae & 2 & 4 & 5 & 10 & 0.29 & - & - & - & - & - & - & - & - & - & - \\
\hline Taeniopterygidae & - & - & - & - & - & 30 & - & - & - & - & 1 & - & - & - & - \\
\hline Philopotomatidae & - & - & - & - & - & 1 & 7 & 5 & 5 & 0.02 & - & - & - & - & - \\
\hline Oligochaeta & - & - & - & - & - & 5 & 1 & 10 & 50 & 0.19 & - & - & - & - & - \\
\hline Libellulidae & - & - & - & - & - & 1 & 6 & 7 & 7 & 0.03 & - & - & - & - & - \\
\hline Corydalidae & - & - & - & - & - & 1 & 6 & 7 & 7 & 0.03 & - & - & - & - & - \\
\hline Leptophlebiidae & - & - & - & - & - & 1 & 8 & 5 & 5 & 0.02 & 5 & 8 & 5 & 25 & 0.23 \\
\hline Psephenidae & - & - & - & - & - & 6 & 7 & 4 & 24 & 0.09 & - & - & - & - & - \\
\hline Hydropsychidae & - & - & - & - & - & 4 & 5 & 5 & 20 & 0.07 & - & - & - & - & - \\
\hline Gyrinidae & - & - & - & - & - & 2 & 4 & 3 & 6 & 0.02 & - & - & - & - & - \\
\hline Elmidae & - & - & - & - & - & 1 & 5 & 4 & 4 & 0.01 & - & - & - & - & - \\
\hline Ferrissidae & - & - & - & - & - & 1 & 3 & 4 & 4 & 0.01 & - & - & - & - & - \\
\hline Dipseudopsidae & - & - & - & - & - & - & - & - & - & - & 1 & - & - & - & - \\
\hline Molaniadae & - & - & - & - & - & - & - & - & - & - & 2 & - & - & - & - \\
\hline Total (T) & 33 & 47 & & & 6.00 & 270 & 67 & & & 5.11 & 109 & 23 & & & 6.75 \\
\hline BMWPICR total & & & & & & & & 99 & & & & & & & \\
\hline IBF/SV2010 total & & & & & & & & 5.9 & & & & & & & \\
\hline Abd total & & & & & & & & 412 & & & & & & & \\
\hline
\end{tabular}


Tabla 7. Tercer muestreo biológico realizado.

\begin{tabular}{|c|c|c|c|c|c|c|c|c|c|c|c|c|c|c|c|}
\hline Mu & & & & & 00 & 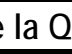 & & ds & t & Hor & n & ii & & 2 2 0 & ) \\
\hline & & untc & $1 \mathrm{El}$ & I Tún & & & unto & $2 \mathrm{P}$ & Puent & & & Pun & to 3 & Final & \\
\hline & & & IBF & F-SV2 & 2010 & & & IBF & $=-S V 2$ & 2010 & & & & F-SV2 & 2010 \\
\hline 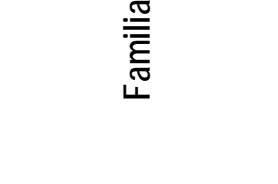 & 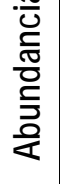 & 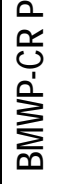 & 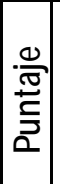 & $\begin{array}{l}\text { : } \\
\frac{7}{*} \\
\frac{0}{0} \\
\text { 운 }\end{array}$ & 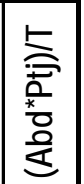 & 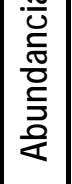 & 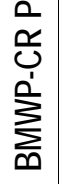 & 离 & 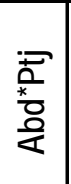 & 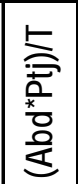 & 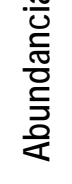 & 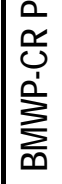 & 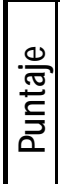 & 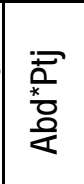 & 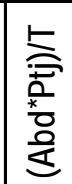 \\
\hline Simuliidae & 78 & 4 & 6 & 468 & 4.73 & 122 & 4 & 6 & 732 & 3.83 & - & - & - & - & ]- \\
\hline Chironomidae & 3 & 2 & 8 & 24 & 0.24 & 7 & 2 & 8 & 56 & 0.29 & 229 & 2 & 8 & 1832 & 7.33 \\
\hline Baetidae & 5 & 5 & 6 & 30 & 0.30 & 30 & 5 & 6 & 180 & 0.94 & - & - & - & - & - \\
\hline Caenidae & 1 & 4 & 7 & 7 & 0.07 & 8 & 4 & 7 & 56 & 0.29 & 6 & 4 & 7 & 42 & 0.17 \\
\hline Gyrinidae & 1 & 4 & 3 & 3 & 0.03 & - & - & - & - & - & - & - & - & - & - \\
\hline Corydalidae & 1 & 6 & 7 & 7 & 0.07 & - & - & - & - & - & - & - & - & - & - \\
\hline Corduliidae & 1 & 8 & 1 & 1 & 0.01 & - & - & - & - & - & - & - & - & - & - \\
\hline Perlidae & 1 & 9 & 2 & 2 & 0.02 & - & - & - & - & - & - & - & - & - & - \\
\hline Hydrophiliadae & 5 & 3 & 7 & 35 & 0.35 & - & - & - & - & - & - & - & - & - & - \\
\hline Coenagrionidae & 1 & 4 & 9 & 9 & 0.09 & - & - & - & - & - & - & - & - & - & - \\
\hline Polycentriopidae & 2 & 6 & 3 & 6 & 0.06 & - & - & - & - & - & - & - & - & - & - \\
\hline Cordulegastridae & - & - & - & - & - & 3 & 8 & 2 & 6 & 0.03 & 2 & 8 & 4 & 8 & 0.03 \\
\hline Gerridae & - & - & - & - & - & 9 & - & 6 & 54 & 0.28 & - & - & - & - & - \\
\hline Psychodidae & - & - & - & - & - & 1 & 3 & 7 & 7 & 0.04 & - & - & - & - & - \\
\hline Potamanthidae & - & - & - & - & - & 8 & 4 & 0 & 0 & 0.00 & - & - & - & - & - \\
\hline Dytiscidae & - & - & - & - & - & 2 & 7 & 7 & 14 & 0.07 & 1 & 4 & 7 & 7 & 0.03 \\
\hline Philopotamidae & - & - & - & - & - & 1 & - & 5 & 5 & 0.03 & - & - & - & - & - \\
\hline Culicidae & - & - & - & - & - & - & - & - & - & - & 7 & 2 & 10 & 70 & 0.28 \\
\hline Physidae & - & - & - & - & - & - & - & - & - & - & 3 & 3 & 3 & 9 & 0.04 \\
\hline Carabidae & - & - & - & - & - & - & - & - & - & - & 2 & . & - & - & - \\
\hline Total $(\mathrm{T})$ & 99 & 55 & & & 5.98 & 191 & 37 & & & 5.81 & 250 & 23 & & & 7.87 \\
\hline BMWPICR total & & & & & & & & 8 & & & & & & & \\
\hline IBF/SV2010 total & & & & & & & & 0.4 & & & & & & & \\
\hline Abd total & & & & & & & & 44 & & & & & & & \\
\hline
\end{tabular}

Fuentes: datos propios y comparados con La Gaceta (2007) y Sermeño et al. (2010). Nota: se observan abreviaturas para abundancia (Abj), puntaje (Ptj). Las casillas color anaranjado corresponden al resultado del índice por punto de muestreo. 


\section{Análisis de cada muestreo:}

Se realizó un análisis general de los índices biológicos de la quebrada El Horno. Se hizo la sumatoria completa de las familias y se obtuvieron los siguientes resultados:

Al observar la Figura 5, en donde se presentan los 2 índices biológicos usados en esta investigación, se puede decir que el punto con mayor contaminación es el punto 3 , ya que la mayor parte de los contaminantes de los puntos de la parte alta (punto 1) y media (punto 2) son transportados hasta este sitio. Para el caso del punto 3 , una de las familias más encontradas en el análisis fue la Chironomidae (Tabla 8), en ambos índices biológicos, esta familia indica estadios de contaminación.

\section{Gráfico 3. Media de los resultados de índices biológicos.}

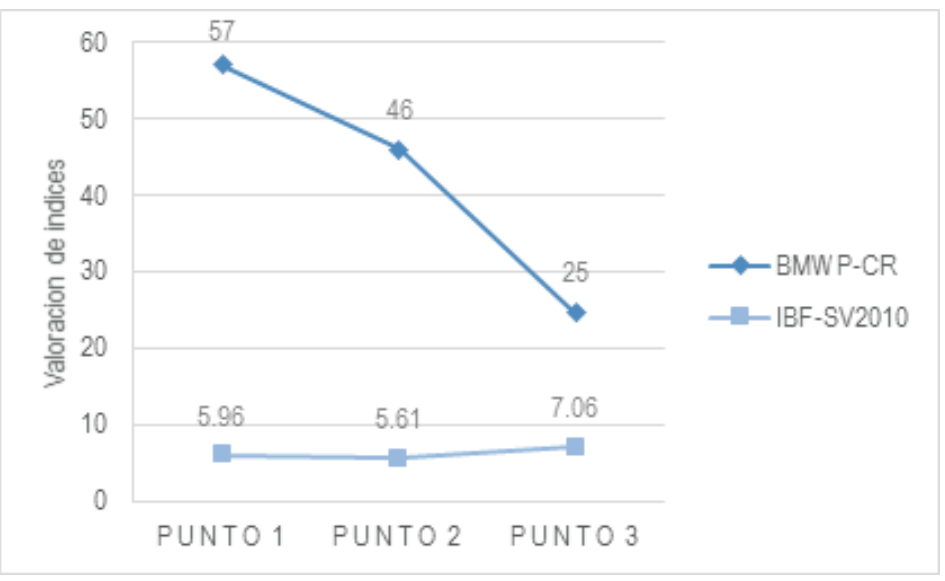

Fuente: datos propios.

Los puntos 1 y 2 tienen niveles de contaminación muy similares (ver Gráfico 3.). Se ha determinado, según los rangos de la Tabla 2, que el agua es de mala calidad (BMWP-CR) y de regular a pobre (IBF-SV2010). Los indicadores biológicos, al igual que los fisicoquímicos y los microbiológicos, demuestran punto por punto la gravedad de la contaminación de la quebrada El Horno.

En la Tabla 8 podemos ver que el valor de BMWP-CR es de 173, y en vista que es mayor que 120, de la Tabla 2 se determinó que el agua es de excelente calidad. Sin embargo, el valor de IBF-SV2010 es de 6.18 y debido a que se localiza en el rango de 5.76 a 6.50 de la Tabla 2, se determinó que el agua es de regular a pobre, con una contaminación sustancialmente probable. 
Tabla 8. Cuantificación total de las familias de la quebrada El Horno.

\begin{tabular}{|c|c|c|c|c|c|c|c|c|c|c|c|}
\hline \multicolumn{12}{|c|}{ Familias encontradas durante los muestreos biologicos de la Quebrada El Horno } \\
\hline \multirow[b]{2}{*}{ 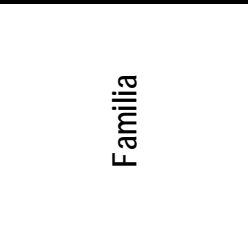 } & \multirow[b]{2}{*}{ 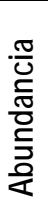 } & \multirow[b]{2}{*}{ 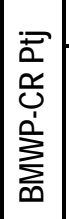 } & \multicolumn{3}{|c|}{ IBF-SV2010 } & \multirow[b]{2}{*}{ 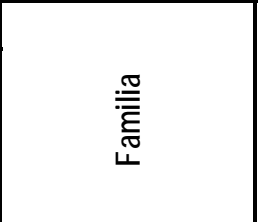 } & \multirow[b]{2}{*}{ 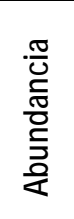 } & \multirow[b]{2}{*}{ 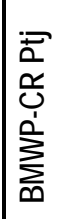 } & \multicolumn{3}{|c|}{ IBF-SV2010 } \\
\hline & & & & $\begin{array}{l}\text { 竞 } \\
\frac{0}{0} \\
\frac{0}{0}\end{array}$ & 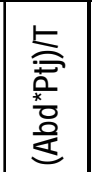 & & & & 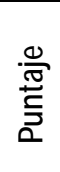 & 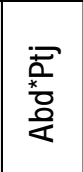 & 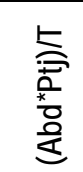 \\
\hline Empididae & 1 & 4 & 6 & 6 & 0.004 & Syrphidae & 3 & 1 & 10 & 30 & 0.021 \\
\hline Ecnomidae & 1 & 10 & - & - & - & Gyrinidae & 3 & 4 & 3 & 9 & 0.006 \\
\hline Haliplidae & 1 & 4 & - & - & - & Physidae & 3 & 3 & 3 & 9 & 0.006 \\
\hline Nepidae & 1 & 4 & 5 & 5 & 0.004 & Platycnemididae & 4 & - & 1 & 4 & 0.003 \\
\hline Helicopsychidae & 1 & 5 & 5 & 5 & 0.004 & Hydropsychidae & 4 & 5 & 5 & 20 & 0.014 \\
\hline Elmidae & 1 & 5 & 4 & 4 & 0.003 & Dytiscidae & 5 & 4 & 7 & 35 & 0.025 \\
\hline Ferrissidae & 1 & 3 & 4 & 4 & 0.003 & Corydalidae & 5 & 6 & 7 & 35 & 0.025 \\
\hline Dipseudopsidae & 1 & - & - & - & - & Oligochaeta & 5 & 1 & 10 & 50 & 0.036 \\
\hline Corduliidae & 1 & 8 & 1 & 1 & 0.001 & Hydrophilidae & 5 & 3 & 7 & 35 & 0.025 \\
\hline Perlidae & 1 & 9 & 2 & 2 & 0.001 & Corduligastridae & 5 & 8 & 2 & 10 & 0.007 \\
\hline Coenagrionidae & 1 & 4 & 9 & 9 & 0.006 & Leptopglebiidae & 6 & 8 & 5 & 30 & 0.021 \\
\hline Phychodidae & 1 & 3 & 7 & 7 & 0.005 & Veliidae & 7 & - & 5 & 35 & 0.025 \\
\hline Glossosomatidae & 2 & 10 & 1 & 2 & 0.001 & Ceratopogonidae & 8 & 4 & 8 & 64 & 0.046 \\
\hline Siphlonuridae & 2 & - & - & - & - & Brachycentridae & 8 & - & - & - & - \\
\hline Scirtidae & 2 & 4 & 6 & 12 & 0.009 & Psephenidae & 8 & 7 & 4 & 32 & 0.023 \\
\hline Planariidae & 2 & 0 & 5 & 10 & 0.007 & Culicidae & 9 & 2 & 10 & 90 & 0.064 \\
\hline Libellulidae & 2 & 6 & 7 & 14 & 0.010 & Gerridae & 15 & 0 & 5 & 75 & 0.053 \\
\hline Tipulidae & 2 & 4 & 5 & 10 & 0.007 & Taeniopterygidae & 31 & - & - & - & - \\
\hline Philopotamidae & 2 & 7 & 5 & 10 & 0.007 & Potamanthidae & 34 & - & - & - & - \\
\hline Molanidae & 2 & - & - & - & - & Caenidae & 44 & 4 & 7 & 308 & 0.220 \\
\hline Polycentripolidae & 2 & 6 & 3 & 6 & 0.004 & Baetidae & 221 & 5 & 6 & 1326 & 0.946 \\
\hline Carabidae & 2 & - & - & - & - & Chironomidae & 373 & 2 & 8 & 2984 & 2.128 \\
\hline Hydroptilidae & 3 & 6 & 4 & 12 & 0.009 & Simuliidae & 560 & 4 & 6 & 3360 & 2.397 \\
\hline Totales (1) & 35 & 102 & & & 0.085 & Totales (2) & 1366 & 71 & & & 6.092 \\
\hline $\begin{array}{l}\Sigma \text { Totales } \\
\text { BMWPICR }\end{array}$ & & & & & & 173 & & & & & \\
\hline $\begin{array}{c}\Sigma \text { Totales } \\
\text { IBF/SV2010 }\end{array}$ & & & & & & 6.18 & & & & & \\
\hline $\begin{array}{l}\Sigma \text { Totales de } \\
\text { Abundancia }\end{array}$ & & & & & & 1401 & & & & & \\
\hline
\end{tabular}

Fuentes: datos propios y comparados con La Gaceta (2007) y Sermeño et al. (2010). Nota: se observan abreviaturas para abundancia(Abj), puntaje (Ptj). 
En general, para el estudio biológico de las microcuencas en la ciudad de Danlí, se recomienda el uso del IBF-SV2010, ya que éste se adapta al ecosistema de la zona oriental porque las altitudes son similares a las de El Salvador. Además, este índice toma en cuenta la abundancia de cada familia, lo cual es clave por la cantidad de individuos encontrados en cada muestreo.

\section{b. Análisis de las encuestas:}

Se aplicaron un total de 630 encuestas a los pobladores de toda la colonia Nueva Esperanza para saber el uso que los pobladores le dan al agua de la quebrada. Un total de 300 de estas encuestas se aplicaron a lo largo de la rivera de la quebrada El Horno y las demás fueron aplicados en las zonas alejadas de la quebrada.

\section{Gráfico 4. Usos del agua de la quebrada El Horno}

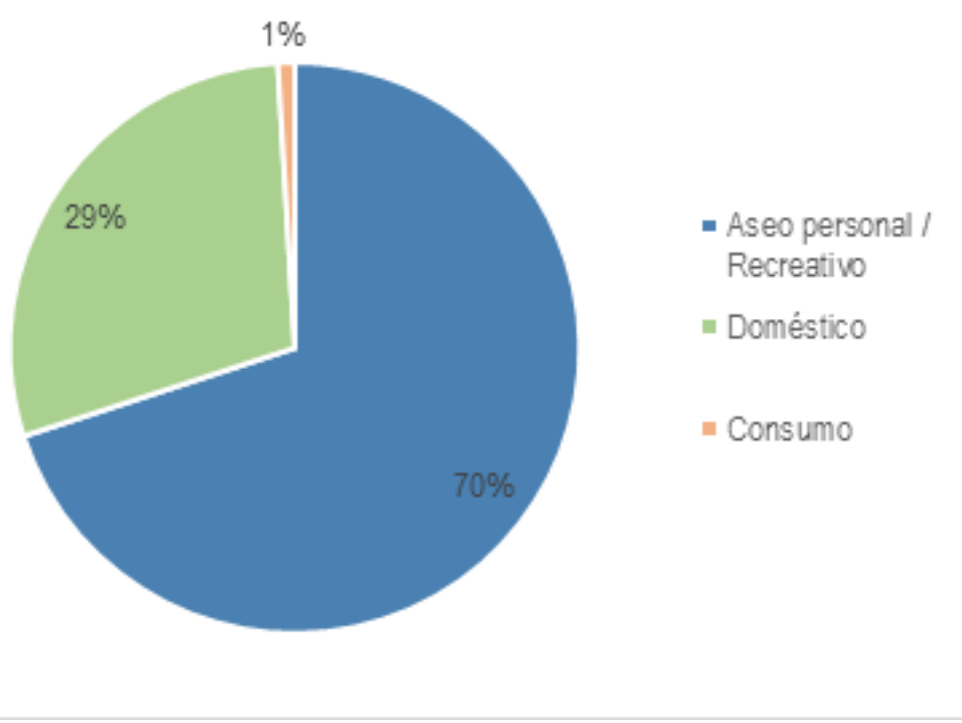

Fuente: datos propios.

Un 63\% de las personas contestó que usaban el agua de la quebrada, 17\% lo usaron alguna vez en la vida y un $20 \%$ nunca la ha usado. Cabe mencionar que de ese $63 \%$ (397 habitantes), el 70\% (441 habitantes) le da un uso para aseo personal y uso recreativo, el 29\% (183 habitantes) de los pobladores lo usan para uso doméstico y un $1 \%$ (6 habitantes) para agua de consumo (ver gráfico 4). 


\section{CONCLUSIONES}

De los 28 análisis fisicoquímicos realizados en la quebrada El Horno de la colonia Nueva Esperanza, se reportan valores elevados en aluminio y hierro, sustancias de uso común como agentes floculantes en los procesos de purificación de agua. La DQO se encontró en cantidades muy grandes, lo que indica el alto consumo de oxígeno que tienen los químicos presentes en la quebrada. Los más altos índices de este parámetro se encontraron en el punto 1.

El análisis microbiológico confirma, en cada una de las muestras tomadas de la quebrada El Horno, que la contaminación a nivel de coliformes totales y fecales es incontable. Esto genera una situación alarmante por la alta cantidad de padecimientos gastrointestinales que se reportan en el centro de salud de la colonia Nueva Esperanza. Los altos niveles de coliformes se deban a la ubicación de los colectores de aguas negras y las tuberías de pozos sépticos que se encuentra descartando desechos a lo largo de la quebrada.

En el caso de los índices biológicos, podemos concluir que el más adecuado para realizar investigaciones en la ciudad de Danlí es el IBF-SV2010, ya que este índice fue desarrollado en un país con características similares a la región oriental (El Salvador). Otra razón que encontramos para ello es que, Por otro lado, este índice considera la abundancia de los individuos. El análisis conjunto de cada punto de la quebrada permite determinar la pobre calidad del agua ya que el BMWP-CR, por sí solo, indica de manera errónea que el agua de la quebrada está en excelentes condiciones. El IBF-SV2010, confirma lo indicado en los análisis químicos y microbiológicos, la existencia de contaminación en el agua de la quebrada El Horno.

\section{RECOMENDACIONES}

Se recomienda la junta de agua y patronato de la colonia Nueva Esperanza la verificación de los desechos que producen las industrias. Además, se debe tomar medidas con los pobladores que tienen tuberías que descargan sus heces fecales, ya que es uno de los resultados que fue más alarmante de esta investigación.

Los pobladores deben saber el peligro que conlleva el uso del agua de la quebrada ya que una buena parte de la población encuestada tiene contacto con el agua de la 
quebrada. Según datos del centro de salud José Celín Discua la mayoría de padecimientos reportados en este centro de salud son por patologías gástricas.

Las autoridades municipales, a través de su unidad municipal ambienta (UMA), deben comenzar a realizar los trabajos de recuperación y restauración de la quebrada El Horno. Además, se debe promover la educación ambiental para los pobladores y específicamente los niños en edad escolar. A los niños se les puede crear una mejor conciencia ambiental y del peligro del uso del agua de la quebrada con estos niveles de contaminación microbiológica y con la presencia de ciertos químicos.

\section{AGRADECIMIENTOS}

Se le agradece a David Martínez, Doctor en Microbiología de UNAH-TEC Danlí el aporte realizado en el análisis de muestras microbiológicas. A los jóvenes Néstor Valladares y Orlando Sotomayor, se les agradece por su ayuda en el manejo de equipo de laboratorio y asistencia técnica en la cuantificación e identificación de macroinvertebrados. De igual manera se les agradece a los alumnos de la carrera de enfermería que cursaron la clase Bl-063 Ecología y Salud II en el tercer periodo académico del 2016 por su ayuda en la recopilación de información y la colecta de muestras químicas, microbiológicas y biológicas en la quebrada El Horno.

\section{REFERENCIAS BIBLIOGRÁFICAS}

Alba, J., \& Sanchez, A. (1988). Un método rápido y simple para evaluar la calidad biológica de las aguas corrientes basado en el de Hellawell (1987). Limnetica 4, 51-56.

Asano, T. (1998). "Wastewater reclamation, recycling and reuse: an introduction. In wastewater reclamation and reuse". Technomic Publishing. Lancaster, 1528.

Barrenechea, A. (2004). ASPECTOS FISICOQUÍMICOS DE LA CALIDAD DEL AGUA. En L. d. Vargas, Tratamiento de agua para Plantas de filtración rápida Manual I: Teoría (págs. 1-55). Lima: OPS/CEPIS/PUB.

Castellon, R. (2013). Evaluación Rápida De La Calidad Del Agua Utilizando Macroinvertebrados Acuáticos Durante La Temporada Lluviosa En La Microcuenca "El Chimbo". Tegucigalpa: Universidad Nacional Autónoma de Honduras.

El Heraldo. (7 de abril de 2014). Vecinos de Danlí se esfuerzan por construir colonias modelo.

(G. Rodriguez, Ed.) Vecinos de Danlí se esfuerzan por construir colonias modelo.

La Gaceta . (17 de Septiembre de 2007). Apendice III: INDICE BMWP-CR. Capitulo V: Del 
monitoreo Biologico, pág. 7.

Ladrera, R., Rieradevall, M., \& Prat, N. (2013). MACROINVERTEBRADOS ACUÁTICOS COMO INDICADORES BIOLÓGICOS: UNA HERRAMIENTA DIDÁCTICA. Revista de Didactica 11, 1-18.

Merritt, R., Cummins, K., \& Berg, M. (2008). An Introduccion to the Aquatic Insects of North America. Kendall/Hunt Publishing Company.

OMS. (2009). Manual para el desarrollo de planes de seguridad del agua. International Water Association.

Rodriguez, X., \& Gentil, M. (2012). Analisis de la calidad del agua de las cuencas "El Gitano"'La Cordillera" del Municipio de Rio de Oro, Cesar mediante el uso del Indice Bmwp (biological monitoring working party) y algunas variables fisicoquimicas . Ocaña: Universidad Francisco De Paula Santander Ocaña .

Roldan, G. (1988). Guía para el estudio de los macroinvertebrados acuáticos del Departamento de Antioquia. Bogota: Universidad de Antioquia.

Secretaria de Salud/CAPRE. (2001). Propuesta nacional de norma para el agua en Honduras. Tegucigalpa: Secretaria de Salud/CAPRE.

Sermeño, J., Serrano, L., Springuer, M., Paniagua, M., Perez, D., Rivas, A., . . Arias, A. (2010). Determinación de la calidad ambiental de las aguas de los ríos de El Salvador, utilizando invertebrados acuáticos: índice biológico a nivel de familias de invertebrados acuáticos en El Salvador (IBF-SV-2010). San Salvador: Universidad de El Salvador . 